\title{
Ferumoxytol-enhanced cardiovascular magnetic resonance detection of early stage acute myocarditis
}

\author{
Yuko Tada, Atsushi Tachibana, Shahriar Heidary, Phillip C. Yang, Michael V. McConnell and Rajesh Dash(D)
}

\begin{abstract}
Background: The diagnostic utility of cardiovascular magnetic resonance (CMR) is limited during the early stages of myocarditis. This study examined whether ferumoxytol-enhanced CMR (FE-CMR) could detect an earlier stage of acute myocarditis compared to gadolinium-enhanced CMR.

Methods: Lewis rats were induced to develop autoimmune myocarditis. CMR (3 T, GE Signa) was performed at the early- (day $14, n=7$ ) and the peak-phase (day $21, n=8$ ) of myocardial inflammation. FE-CMR was evaluated as $\%$ myocardial dephasing signal loss on gradient echo images at 6 and $24 \mathrm{~h}$ ( $6 \mathrm{~h}$ - \& $24 \mathrm{~h}$-FE-CMR) following the

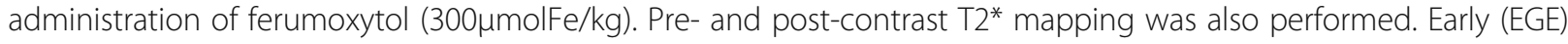
and late (LGE) gadolinium enhancement was obtained after the administration of gadolinium-DTPA $(0.5 \mathrm{mmol} / \mathrm{kg})$ on day 14 and 21 . Healthy rats were used as control $(n=6)$.
\end{abstract}

Results: Left ventricular ejection fraction (LVEF) was preserved at day 14 with inflammatory cells but no fibrosis seen on histology. EGE and LGE at day 14 both showed limited myocardial enhancement (EGE: $11.7 \pm 15.5 \%$; LGE: $8.7 \pm 8.7 \%$; both $p=$ ns vs. controls). In contrast, 6 h-FE-CMR detected extensive myocardial signal loss (33.2 $\pm 15.0 \%$, $p=0.02$ vs. EGE and $p<0.01$ vs. LGE). At day 21 , LVEF became significantly decreased (47.4 $\pm 16.4 \%$ vs control: $66.2 \pm 6.1 \%$, $p<0.01)$ with now extensive myocardial involvement detected on EGE, LGE, and $6 \mathrm{~h}-\mathrm{FE}-\mathrm{CMR}(41.6 \pm 18.2 \%$ of LV). T2* mapping also detected myocardial uptake of ferumoxytol both at day $14\left(6 \mathrm{~h} \mathrm{R} 2^{*}=299 \pm 112 \mathrm{~s}^{-1}\right.$ vs control: $125 \pm 26 \mathrm{~s}^{-1}$, $p<0.01)$ and day 21 (564 $\pm 562 \mathrm{~s}^{-1}, p<0.01$ vs control). Notably, the myocardium at peak-phase myocarditis also showed significantly higher pre-contrast T2* (27 $\pm 5 \mathrm{~ms}$ vs control: $16 \pm 1 \mathrm{~ms}, p<0.001)$, and the extent of myocardial necrosis had a strong positive correlation with $\mathrm{T} 2^{*}(r=0.86, p<0.001)$.

Conclusions: FE-CMR acquired at $6 \mathrm{~h}$ enhance detection of early stages of myocarditis before development of necrosis or fibrosis, which could potentially enable appropriate therapeutic intervention.

Keywords: Ferumoxytol, CMR, MRI, Myocarditis, T2* map

\section{Subject terms}

Inflammatory heart disease, Cardiovascular Magnetic Resonance Imaging (CMR)

\section{Background}

Myocarditis is reported to account for up to $10-20 \%$ of acute-onset heart failure, sudden death among young adults and athletes, and unexplained cardiomyopathies [1-5]. However, the precise incidence and prevalence of

* Correspondence: rhombus@stanford.edu

Department of Medicine (Cardiovascular Medicine), Stanford University

School of Medicine, 300 Pasteur Drive, Stanford, CA 94305, USA myocarditis remain poorly characterized because of challenges in diagnostic accuracy [6, 7]. Current guidelines recommend the use of cardiovascular magnetic resonance (CMR) and myocardial biopsy for the diagnosis of myocarditis in patients with heart failure [8]. However, the usability of biopsy is limited because of its invasiveness and low sensitivity, with only $40 \%$ sensitivity for myocarditis [9-11]. Instead, non-invasive imaging approaches are used - with varying success - to diagnose inflammatory cardiac diseases, quantify disease activity, guide therapeutic interventions, and predict disease progression.

(c) The Author(s). 2019 Open Access This article is distributed under the terms of the Creative Commons Attribution 4.0 International License (http://creativecommons.org/licenses/by/4.0/), which permits unrestricted use, distribution, and reproduction in any medium, provided you give appropriate credit to the original author(s) and the source, provide a link to the Creative Commons license, and indicate if changes were made. The Creative Commons Public Domain Dedication waiver (http://creativecommons.org/publicdomain/zero/1.0/) applies to the data made available in this article, unless otherwise stated. 
CMR characterizes many of the histological changes caused by myocarditis. Edematous lesions, hyperemia/vascular leakage, and necrotic or scarred tissue can be detected using T2-weighted (T2w) imaging, early gadolinium enhancement (EGE), and late gadolinium enhancement (LGE) imaging, respectively $[12,13]$. The accuracy of combining T2w imaging and LGE in diagnosing myocarditis is reported to be 78\% [14]. However, the specificity of CMR for inflammation is still controversial and the sensitivity for detecting reversible myocardial injury in the early inflammatory phase is relatively low [15]. Global myocardial pathologies such as diffuse inflammation or fibrosis are also difficult to delineate when using CMR techniques that depend on comparison to unaffected myocardium. Although T2w imaging is more specific for active inflammation, interpretation of $\mathrm{T} 2 \mathrm{w}$ imaging tends to be qualitative and remains challenging.

Superparamagnetic iron oxide (SPIO) particles have been used to label myocardial cellular infiltrates to be detected on CMR [16]. SPIO administered intravenously is delivered to the interstitium by non-specific vesicular transport and through trans-endothelial channels, and taken up by inflammatory cells. Because of the superparamagnetic effects of the agents, labeled cells are detected as signal loss on T2\%weighted sequences. A longer half-life in the blood $(15 \mathrm{~h})$ and wider distribution of ultra-small SPIO (USPIO) are advantageous in visualizing inflammatory cellular infiltrates [17]. Ferumoxytol, an FDA-approved USPIO, has been already used clinically as an CMR contrast agent, with rates of adverse event and anaphylaxis reported to be as low as 0.2 and $0.02 \%$, respectively [18]. The enhancement obtained from 6 to $24 \mathrm{~h}$ has been utilized to visualize inflammatory cells in atherosclerosis or myocardial infarction, which correlates with disease activity [19-21]. However, it remains unknown whether ferumoxytol-enhanced CMR (FE-CMR) has higher sensitivity and specificity for the diagnosis of early and active phases of myocarditis compared to gadoliniumenhanced CMR. In the preclinical rodent myocarditis model, detectability of inflammation at specific timepoints can be tested, compared between contrast agents, and verified by histological evaluation accurately. This study adopted the well-established rat autoimmune myocarditis model, which reaches an inflammatory peak at approximately day 21 post immunization and contains similar types of infiltrating cells compared to human myocarditis [22, 23]. We divided the rats into three groups; control without myocarditis, early stage day 14 , and advanced stage day 21 , and we performed FE-CMR, T2* mapping and gadolinium-enhanced CMR at each stage of myocarditis.

\section{Methods}

Rat experimental autoimmune myocarditis model

The animal protocols in this research were approved by the Stanford University Administrative Panel on Laboratory
Animal Care. Male Lewis rats $(n=21,6$ weeks of age, 101$125 \mathrm{~g}$ ) were obtained from Charles River Laboratories (Wilmington, Massachusetts, USA). Rats were fed a standard diet and water and maintained in compliance with animal welfare guidelines of the Institute of Experimental Animals. Experimental autoimmune myocarditis was induced by immunizing rats with purified porcine cardiac myosin (Sigma Aldrich, St. Louis, Missouri, USA). The cardiac myosin was emulsified with complete Freund's adjuvant (DIFCO, Sparks, Maryland, USA) supplemented with Mycobacterium tuberculosis (DIFCO) at a concentration of $10 \mathrm{mg} / \mathrm{ml}$. $0.2 \mathrm{ml}$ of emulsion (immunizing dose of $1.0 \mathrm{mg}$ of cardiac myosin per rat) was injected into the rat footpads subcutaneously [24]. During the procedure, rats were anesthetized by inhaled isoflurane (1.0-3.0\%). For analgesia, buprenorphine $(0.05 \mathrm{mg} / \mathrm{kg})$ was injected subcutaneously before and after the procedure as needed.

\section{CMR}

CMR was performed at $3 \mathrm{~T}$ (Signa EXCITE, General Electric Healthcare, Waukesha, Wisconsin, USA) scanner and a phased array 2 channel surface coil (Rapid MR international, Columbus, Ohio, USA). Rats were anesthetized during the scan using $1.0-3.0 \%$ of isoflurane. CMR was done under the optimized electrocardiogram (ECG) gating monitored and controlled by PC-SAM (SA Instrument, Stoneybrook, New York, USA). Heart rates during the scan were between 300 and 400/min.

The rats were evaluated at early- (day 14) $(n=7)$ and peak-phase (day 21) $(n=8)$ of myocardial inflammation. The study design is shown in Fig. 1. Cardiac function was evaluated by cine-CMR. For cine CMR, FSPGR sequence (flip angle $=45^{\circ}, \mathrm{TE}=10 \mathrm{~ms}, \mathrm{TR}=20 \mathrm{~ms}, \mathrm{NEX}=$ 4 , matrix $=256 \times 192$, FOV $=6-7 \mathrm{~cm}$, thickness $=1.5 \mathrm{~mm}$, $\mathrm{BW}=122 \mathrm{~Hz} /$ pixel, phases to reconstruct $=20$ ) was used to obtain 5-6 slices covering through the base to apex. Multi-slice gradient echo CMR (GRE) images were taken on the left ventricular (LV) short axis planes for T2* mapping. GRE images at different echo times (TE) times $(4.9,6.8,9.0,11.3,13.5,15.8,18.0 \mathrm{~ms})$ were used to acquire multiple short-axis images of the mid LV (flip angle $=35^{\circ}, \mathrm{TR}=1 \mathrm{RR}$, trigger delay $=12 \mathrm{~ms}, \mathrm{NEX}=6$, matrix $=256 \times 192, \quad F O V=4-5 \mathrm{~cm}$, thickness $=1.5 \mathrm{~mm}$, $\mathrm{BW}=122 \mathrm{~Hz} /$ pixel). For gadolinium-enhanced CMR, $0.5 \mathrm{mmol} / \mathrm{kg}$ of Gd-DTPA (Magnevist, Bayer Health Care Pharma AG, Berlin, Germany) was injected through the tail vein, and EGE and LGE were obtained. EGE was obtained with ECG-triggered T1-weighted sequence at the mid LV immediately after the gadolinium injection $(\mathrm{TE}=16 \mathrm{~ms}$, $\mathrm{TR}=1 \mathrm{RR}, \quad \mathrm{NEX}=5, \quad$ matrix $=256 \times 192, \quad \mathrm{FOV}=4-5 \mathrm{~cm}$, thickness $=1.5 \mathrm{~mm}, \mathrm{BW}=61 \mathrm{~Hz} /$ pixel). LGE was evaluated on ECG-triggered IR-FSPGR sequence (flip angle $=30^{\circ}, \mathrm{TE}=$ $4.4 \mathrm{~ms}, \mathrm{TR}=12.9 \mathrm{~ms}, \mathrm{NEX}=5$, matrix $=256 \times 192$, FOV $=4$ $5 \mathrm{~cm}$, thickness $=1.5 \mathrm{~mm}, \mathrm{BW}=61 \mathrm{~Hz} /$ pixel, $\mathrm{TI}=180-200$ 


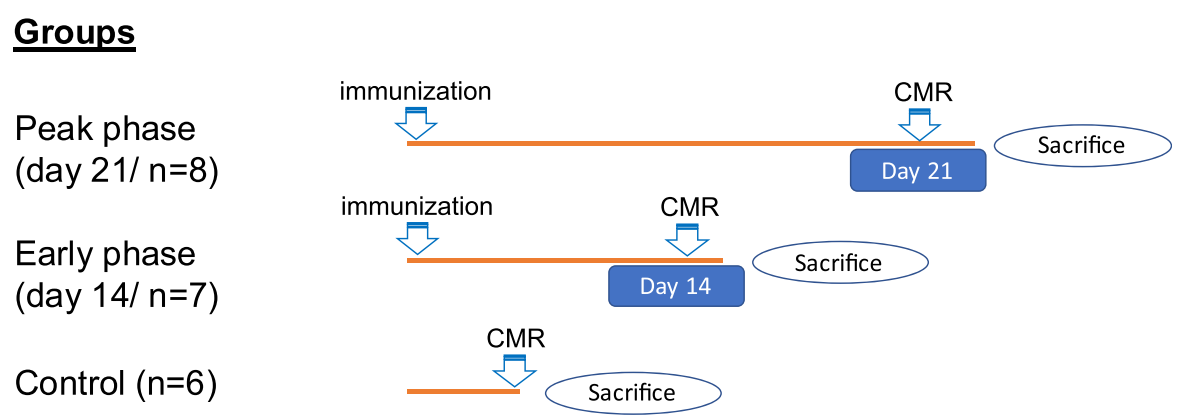

\section{CMR protocol}

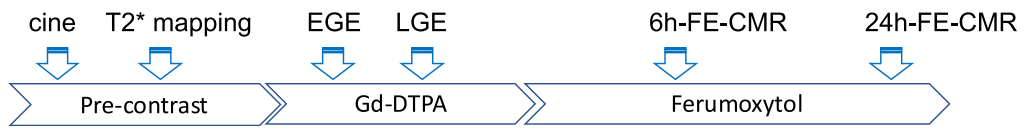

Fig. 1 Study design The schema shows the timeline for each group and the CMR protocol

ms) performed during 10-20 min after the injection of GdDTPA. After gadolinium-enhanced CMR, ferumoxytol (Feraheme; AMAG Pharmaceuticals, Inc. Lexington, Massachusetts, USA) was administered into the rat tail vein or the femoral vein. Ferumoxytol $(300 \mu \mathrm{molFe} / \mathrm{kg})$ was administered based on the manufacture's instruction and the previous reports $[25,26]$. Dephasing signal loss was detected on GRE twice at 6 and $24 \mathrm{~h}$ later for each rat after washout of GdDTPA was verified. The same studies were performed in control rats $(n=6)$ that were not induced with myocarditis.

\section{CMR analysis}

Cine CMR was analyzed using Horos (Annapolis, Maryland, USA). LV endocardium and epicardium on enddiastolic and end-systolic sections were traced manually, and thus LV end-diastolic volume (LVEDV) and LV endsystolic volume (LVESV) were calculated [27]. LVEF was determined according to the formula; LVEF $=($ LVEDV LVESV)/ LVEDV $\times 100(\%)$. LV mass $(L V M)(g)$ was determined as $1.05 \mathrm{~g} / \mathrm{cm}^{3}$ times $\mathrm{LV}$ wall volume. LVM, LVEDV and LVESV were divided by the body weight (BW) to obtain indexed values; LVM/BW, LVEDV/BW, and LVESV/BW, respectively.

The inflammatory area was calculated by Matlab analytic (Mathworks, Natick, Massachusetts, USA). Slices of the mid LV were used to compare image sequences. LV was segmented by tracing epicardial and endocardial contours manually. Mean +/- SD of the signal intensity (SI) was calculated in the remote region of interest (ROI) placed on the skeletal muscle of the chest. All pixels within $\mathrm{LV}$ with $\mathrm{SI}>2 \mathrm{SD}$ higher than the remote ROI was considered positive signal on EGE and LGE images. Negative enhancement with ferumoxytol was evaluated on GRE and myocardium that displayed $<20 \%$ of SI of the remote ROI was considered abnormal signal void. This cut-off was selected based on the previous report and the visual correspondence [28]. The inflammatory area was shown as the percentage of the total LV area. T2* was calculated by applying GRE images taken at different TEs to the $\mathrm{T} 2^{*}$ signal intensity decay formula: $\mathrm{I}_{\mathrm{t}}=\mathrm{I}_{\mathrm{t}=0} \times \mathrm{e}^{-\mathrm{TE} / \mathrm{T} 2^{*}}$ and $\mathrm{T} 2^{*}$ map was created. Then, $\mathrm{T}^{*}$ and $\mathrm{R} 2 *(=1 / \mathrm{T} 2 *)$ of LV were evaluated by tracing LV contours. Finally, the change in R2* of LV, which represents the distribution of ferumoxytol, was determined: delta R2* = R2* (post-contrast)-R2* (pre-contrast).

Contrast to noise ratio (CNR) of EGE, LGE and FECMR were calculated to compare the contrast between the lesion and the non-lesion. This was calculated according to the formula:

$\mathrm{CNR}=\left(\mathrm{S}_{(\text {lesion) }}-\mathrm{S}_{(\text {non-lesion })}\right) / \sigma$.

$\mathrm{S}_{\text {(lesion) }}=$ mean SI of the lesion,

$S($ non-lesion $)=$ mean SI of the non-lesion,

$\sigma_{(\text {lesion })}=$ standard deviation of the lesion.

$\sigma_{(\text {non-lesion })}=$ standard deviation of the non-lesion.

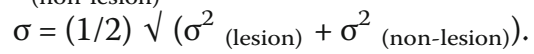

CMR analysis was performed by two researchers and one of them was blinded to the inflammatory phases.

\section{Histology}

After the CMR studies, the rats were euthanized by cutting the aorta under anesthesia ( $5 \%$ isoflurane) at day 14 or day 21 , respectively for each group and the ex-vivo histological evaluation was performed. The removed hearts were cut into halves. One was fixed in $10 \%$ formalin and embedded in paraffin, and the other was embedded in OCT compounds (Sakura Finetek) and snap-frozen. Paraffin-embedded tissues 
were used for staining with Hematoxylin-Eosin (H\&E), Masson's Trichrome, and Prussian blue to evaluate myocardial inflammatory infiltrates/necrosis, myocardial fibrosis, and iron particle accumulation, respectively. The sections were examined and the pictures were taken using BZ-X700 microscope and its internal software (Keyence Corporation of America, Itasca, Illinois, USA). Areas with inflammatory cellular infiltrates were traced on $\mathrm{H} \& \mathrm{E}$ staining and the ratio to the LV was analyzed using ImageJ. Nuclei were highlighted by the threshold to detect nuclei of myocardium clearly on color-split images. The area with clusters of infiltrating cells were enclosed by polygons (Fig. 2). Thus, the total inflammatory areas were calculated. Fibrosis and necrosis were quantified on Masson Trichrome staining using the software calculated on Matlab. The RGB images were converted to LAB color space and the specific colors of fibrosis or necrotic tissue were selected by placing ROI in representative fibrotic or necrotic regions, respectively. Then, for each pixel, the difference between the pixel's color and the average LAB color of selected region was calculated. The threshold was set to minimize the overlapping of the colors of segmented regions. Thus, fibrotic and necrotic regions were segmented and calculated (Fig. 2). Ferumoxytol particles distributed in the non-necrotic or necrotic inflammatory regions were counted under high-power field $(\times 400)$ of Prussian blue staining slides. The numbers of clearly identifiable intracellular ferumoxytol particles contained in 3 different locations of the non-necrotic or necrotic myocardial inflammation per each rat were summed respectively and analyzed. Frozen tissues were sectioned at the thickness of $7 \mathrm{um}$, fixed with $4 \%$ paraformaldehyde, blocked with $3 \%$ hydrogen peroxide, and incubated with anti-CD68 (ED1) antibody (mouse monoclonal, abcam, Cambridge, Massachusetts, USA) at $4{ }^{\circ} \mathrm{C}$ for overnight. Antibody-HRP conjugate was detected with a Histofine Simplestain system (Nichirei Corporation, Tokyo, Japan) and visualized with DAB substrates. Sections were counterstained with hematoxylin. The number of infiltrating CD68 positive cells were counted on the pictures taken under high magnification $(\times 100)$. Ten slices to cover the entire heart were used for each rat ( $n=5$ each for day 21 and day 14 group (50 slices per group) and $n=3$ for control (30 slices)). The average numbers of infiltrating macrophages per slice were determined. To detect colocalization of macrophage, sections were stained with Prussian blue following immunohistochemistry for CD68 and nuclei were stained with methyl green. Slices obtained at both 6 and $24 \mathrm{~h}$ postferumoxytol injection were used ( $n=3$ in each group). Histological analysis was performed by two researchers and one of them was blinded to the inflammatory phases.

\section{The Miles assay}

The enhanced vascular permeability and retention (EPR) effect in the rats at day $14(\mathrm{n}=3)$ was evaluated by the Miles assay [29]. In this assay, pathological increase of vascular permeability can be assessed by extravasation of Evans Blue bound to albumin, which is impermeable to the endothelium under normal physiological condition. Evans Blue dissolved in saline $(30 \mathrm{mg} / \mathrm{kg})$ was injected through the tail vein and $30 \mathrm{~min}$ later, the rats were

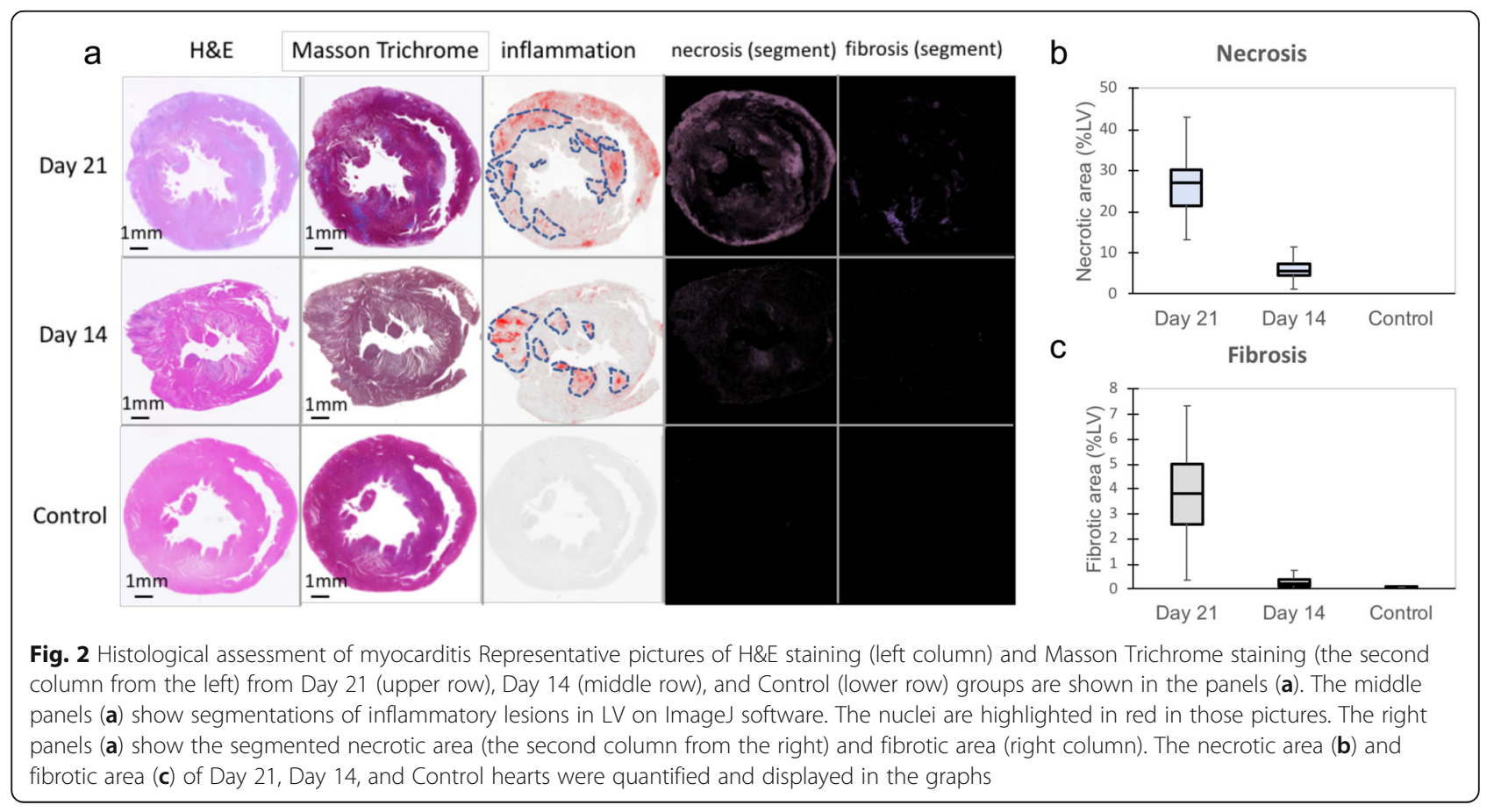


perfused systemically with saline to wash out Evans Blue dye from the blood. The hearts were sliced and the pictures were taken to assess extravasation of Evans Blue visually.

\section{Statistical analysis}

All data were expressed as mean \pm SD. XLSTAT software (Addinsoft, Paris, France) was used for statistical analysis. The normal distribution of the data was confirmed using Shapiro-Wilk test. Means between two groups were compared using a two tailed t-test for the data with normal distribution. Non-parametric analysis (Mann-Whitney test, two-tailed) were used for the data without normal distribution. Differences with values of $p<0.05$ were considered significant. Pearson's correlation coefficient $(r)$ was determined to test linear correlation between two sample sets.

\section{Results}

\section{Development of autoimmune myocarditis}

Myocarditis was confirmed histologically in all rats at both days 14 and 21 following the immunization, with inflammatory cellular infiltration quantified as $26.6 \pm$ $8.7 \%$ of LV at day 14 and $44.5 \pm 17.5 \%$ of LV at day 21 (day 14 vs day $21, p=0.03$ ) (Fig. 2). Visually, myocardial necrosis was subtle with no fibrotic replacement found at day 14. However, inflammatory regions at day 21 showed multiple myocardial necrotic foci. The size of necrotic region was calculated as $26.3 \pm 9.3 \%$ of $\mathrm{LV}$ at day 21 and $6.4 \pm 3.5 \%$ of LV at day 14 ( $p<0.001$ vs day $21)$. Fibrosis was quantified as $3.7 \pm 2.5 \%$ of $L V$ at day 21 ( $p=0.003$ vs day $14, p=0.004$ vs control), $0.29 \pm 0.25 \%$ of LV at day 14 ( $p=0.04$ vs control), and $0.056 \pm 0.028 \%$ of $\mathrm{LV}$ in the control. The degree of necrosis and fibrosis in myocarditis at days 14 and 21 had a positive correlation $(r=0.85, p<0.001)$. The LV functional analysis is shown in Table 1 . At day 14, LVM/BW was mildly increased $(2.22 \pm 0.54 \mathrm{mg} / \mathrm{g}$ vs the control; $1.46 \pm 0.27 \mathrm{mg} / \mathrm{g}$, $p<0.01$ ) but LV function was preserved (LVEF; $64.6 \pm$ $7.7 \%$, vs control $66.2 \pm 6.1 \%$ ). Conversely, at the peak stage of day $21, \mathrm{LVM} / \mathrm{BW}$ was more substantially increased in the myocarditis group (LVM/BW $3.01 \pm 0.99 \mathrm{mg} / \mathrm{g}, p<$ 0.01 vs control) and LV function was significantly decreased (LVEF; $47.4 \pm 16.4 \%, p<0.01$ vs control).

\section{CMR detection of myocardial inflammation}

Representative CMR images of each group are shown in Fig. 3a. The region of myocardial inflammation on each image modality was calculated (Fig. 3b). The SI of the remote ROIs in the skeletal muscle showed comparable values between the control and the EAM groups. At the early phase of day 14, 6 h-FE-CMR could clearly detect the dephasing signal loss in the inflammatory lesions with an average inflammatory area of $33.2 \pm 15.0 \%$, which corresponded well with histological inflammatory
Table 1 LV functional analysis

\begin{tabular}{llll}
\hline & Control & Day 14 & Day 21 \\
\hline LVEF $(\%)$ & $66(6)$ & $65(8)$ & $47(16) \dagger$ \\
LVM $(\mathrm{mg})$ & $345(33)$ & $348(72)$ & $545(156) *$ \\
LVM/BW $(\mathrm{mg} / \mathrm{g})$ & $1.46(0.27)$ & $2.22(0.54) \dagger$ & $3.01(0.99) \dagger$ \\
LVEDV $(\mu \mathrm{l})$ & $361(34)$ & $245(37) \dagger$ & $309(22) \dagger$ \\
LVEDV/BW $(\mu \mathrm{l} / \mathrm{g})$ & $1.52(0.24)$ & $1.56(0.22)$ & $1.69(0.15)$ \\
LVESV $(\mu \mathrm{l})$ & $121(16)$ & $86(17) \dagger$ & $163(55)$ \\
LVESV/BW $(\mu \mathrm{l} / \mathrm{g})$ & $0.50(0.02)$ & $0.54(0.13)$ & $0.90(0.33) \dagger$ \\
BW $(\mathrm{g})$ & $239(25)$ & $158(14) \dagger$ & $183(15) \dagger$ \\
\hline
\end{tabular}

LV function was analyzed on cine CMR. Mean (SD) of LVEF, LVM, LVM/BW, LVEDV, LVEDV/BW, LVESV, LVESV/BW, and BW of the control, early phase (day 14 ), and peak phase (day 21) groups are shown. [BW = body weight. Statistical analysis; ${ }^{*} p=0.01,+p<0.01$ compared to the control.] LVEDV, left ventricular end-diastolic volume; LVEF, left ventricular ejection fraction; LVESV, left ventricular end-systolic volume; LVM, left ventricular mass

infiltration. This decreased to $15.4 \pm 13.5 \%$ on $24 \mathrm{~h}$-FECMR $(p=0.03$ vs 6 h). EGE $(11.7 \pm 15.5 \%, p=0.02)$ and LGE $(8.7 \pm 8.7 \%, p<0.01)$ on day 14 demonstrated significantly decreased detection of myocarditis, which was not significantly different from the areas calculated as false positive in the control: EGE $4.1 \pm 2.1 \%$, LGE $4.1 \pm$ $2.1 \%$, and FE-CMR $4.0 \pm 3.2 \%$ ( $p=0.001$ compared to 6 h-FE-CMR). EGE, LGE and FE-CMR had comparable CNR: $3.94 \pm 0.50$ (EGE), $4.38 \pm 0.35$ (LGE), and $3.94 \pm$ 0.37 (FE-CMR) $(p>0.05)$.

At the peak inflammatory phase of day 21, FE-CMR performed at $6 \mathrm{~h}$ after the ferumoxytol injection $(6 \mathrm{~h}-\mathrm{FE}$ CMR) showed extensive and distinct signal loss. Inflammatory area detected on $6 \mathrm{~h}-\mathrm{FE}-\mathrm{CMR}$ was $41.6 \pm 18.2 \%$, which showed a larger trend compared to EGE (31.5 \pm $15.7 \%$, ns $)$ and LGE $(27.2 \pm 12.0 \%$, ns). The areas with signal loss detected on $6 \mathrm{~h}$-FE-CMR were significantly decreased, though still detectable, at $24 \mathrm{~h}$ (24h-FECMR) $(10.9 \pm 11.5 \%, p=0.02$ vs. $6 \mathrm{~h})$.

Figure 4 and Table 2 show the pre- and post-contrast $\mathrm{T}^{*}$ and delta R2* of each group. T2* of LV significantly decreased at $6 \mathrm{~h}$-post-contrast both at days 14 and 21 compared to the control. Delta R2* of LV demonstrated the significantly enhanced uptake of ferumoxytol $6 \mathrm{~h}$ post-contrast both at day $14\left(299 \pm 112 \mathrm{~s}^{-1}, p<0.01\right)$ and day $21\left(564 \pm 562 \mathrm{~s}^{-1}, p<0.01\right)$ compared to the control $\left(125 \pm 26 \mathrm{~s}^{-1}\right)$. On the other hand, while significant difference in delta $\mathrm{R} 2 *$ at 24 h-post contrast compared to the control $\left(46 \pm 19 \mathrm{~s}^{-1}\right)$ were observed at day $14\left(89 \pm 18 \mathrm{~s}^{-1}, p<0.01\right)$, significant difference in delta $\mathrm{R} 2^{*}$ was not confirmed at day $21\left(77 \pm 39 \mathrm{~s}^{-1}\right)$.

Native $\mathrm{T} 2 *$ of the LV was significantly increased at day 21 compared to control (day $21 ; 27 \pm 5 \mathrm{~ms}$ vs control; $16 \pm 1 \mathrm{~ms}, p<0.001$ ), however, no significant change was observed at day $14(17 \pm 3 \mathrm{~ms})$. Histologically, areas with high $\mathrm{T}^{*}$ values corresponded with advanced inflammation accompanying necrosis (Fig. 4c). The quantified 

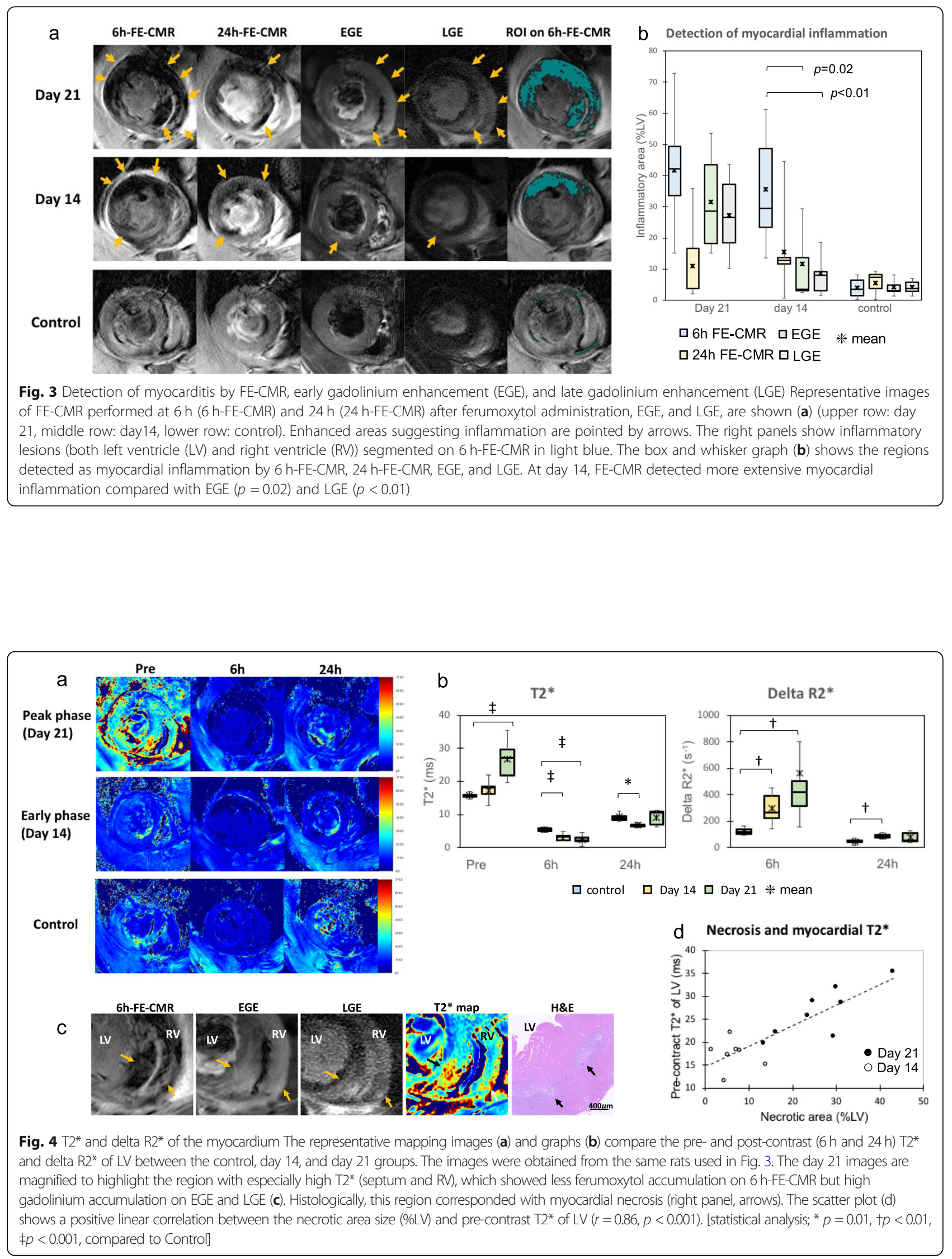
Table $2 \mathrm{~T}^{\mathrm{a}}$ and delta $\mathrm{R} 2^{\mathrm{a}}$ of myocardium

\begin{tabular}{lllll}
\hline & & Control & Day 14 & Day 21 \\
\hline T2 $^{*}(\mathrm{~ms})$ & Pre & $15.9(0.5)$ & $17.3(1.2)$ & $26.7(1.9) \neq$ \\
& $6 \mathrm{~h}$ & $5.3(0.2)$ & $3.0(0.3) \neq$ & $2.4(0.2) \neq$ \\
& $24 \mathrm{~h}$ & $9.4(0.7)$ & $6.7(0.2)^{*}$ & $9.1(1.0)$ \\
Delta R2* $\left(\mathrm{s}^{-1}\right)$ & $6 \mathrm{~h}$ & $125(10.5)$ & $299(42.4) \dagger$ & $564(198.5) \dagger$ \\
& $24 \mathrm{~h}$ & $46(8)$ & $89(7) \dagger$ & $77(17)$
\end{tabular}

T2* and delta R2* of LV obtained pre, and $6 \mathrm{~h}$ and $24 \mathrm{~h}$ post ferumoxytol injection are shown (control, day 14, and day 21 groups). [statistical analysis; * $p=0.01,+p<0.01, \neq p<0.001$, compared to Control]

necrotic regions had a positive linear correlation with myocardial pre-contrast $\mathrm{T}^{*}(r=0.86, p<0.001)$ (Fig. $4 \mathrm{~d})$. The quantified fibrotic regions also had a positive linear correlation with $\mathrm{T} 2 *(r=0.82, p<0.001)$. FE-CMR showed decreased negative contrast effects in areas with increased native $\mathrm{T} 2 *$ corresponding with myocardial necrosis whereas EGE and LGE showed strong positive contrast in those regions (Fig. 4c).

\section{Iron distribution in the necrotic/ non-necrotic regions}

Iron staining with Prussian blue also confirmed that ferumoxytol distributed in the regions with inflammatory cellular infiltration. The inflammatory cellular uptake of ferumoxytol particles was observed in the ex-vivo hearts obtained at $24 \mathrm{~h}$ after the ferumoxytol injection. However, the necrotic and non-necrotic inflammation revealed distinctively different distribution of ferumoxytol. Inflammatory tissues with myocardial necrosis contained fewer iron particles while non-necrotic inflammatory tissues showed prominent distribution of iron particles (Fig. 5). Quantification of iron particles demonstrated significantly fewer iron particles in the necrotic tissues (7.2 \pm 6.5 particles /high power field) compared to non- necrotic inflammatory tissues $(98.3 \pm 39.6$ particles /high power field, $p<0.001$ ) (Fig. 5).

\section{Iron uptake by macrophages}

Macrophage infiltration was confirmed in both Day 14 and 21 EAM hearts (Fig. 6). Day 21 heart had more extensive infiltration of CD68 positive macrophages compared to Day 14 heart $(767 \pm 201$ counts/field vs $282 \pm$ 120 counts/field, $p<0.0001)$. CD68 positive macrophages were scarcely found in the normal control heart $(2.8 \pm 2.7$ counts/field). Prussian blue staining and immunohistochemistry showed co-localization of ferumoxytol in some of the CD68 positive macrophages at $24 \mathrm{~h}$ postferumoxytol injection (Fig. 6). In contrast, Prussian blue staining and immunohistochemistry at $6 \mathrm{~h}$ postferumoxytol injection confirmed ferumoxytol distribution in the extracellular space adjacent to the infiltrating macrophages (Fig. 6).

\section{Enhanced vascular permeability and FE-CMR}

The dependence of distribution of ferumoxytol on the enhanced vascular permeability was investigated by visualizing extravasation of Evans Blue in the tissue (Fig. 7). The areas of signal void on $6 \mathrm{~h}$-FE-CMR corresponded with the areas with the myocardium stained blue with Evans Blue's extravagation in the inflammatory tissue.

\section{Discussion}

This study showed $6 \mathrm{~h}$-FE-CMR has an excellent and superior capability to detect early active myocarditis. Most remarkably, FE-CMR demonstrated a higher sensitivity to the early stage of acute myocarditis compared to EGE and LGE. Conversely, FE-CMR was less sensitive to the necrotic regions in the advanced phase of inflammation while EGE, LGE, and $\mathrm{T} 2 \%$ showed high sensitivity to

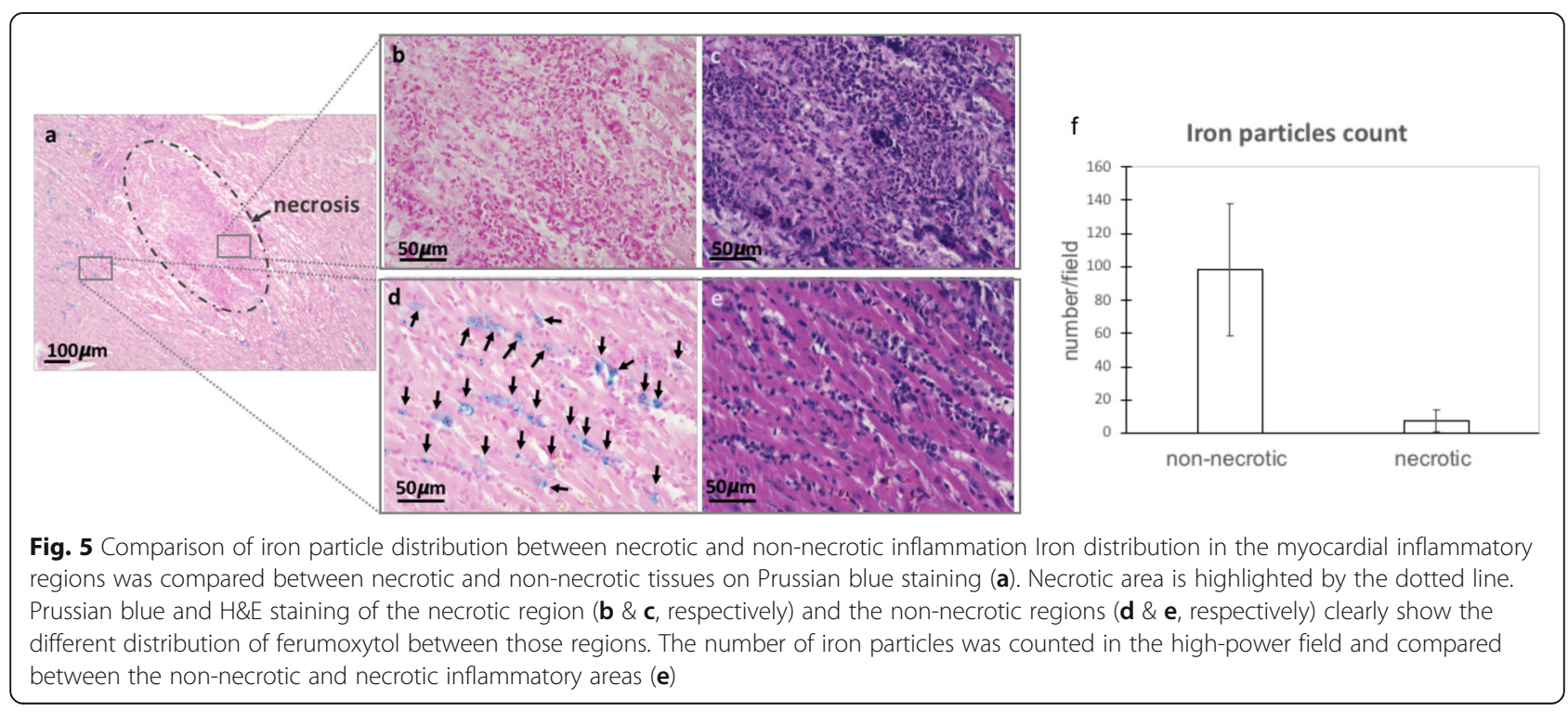




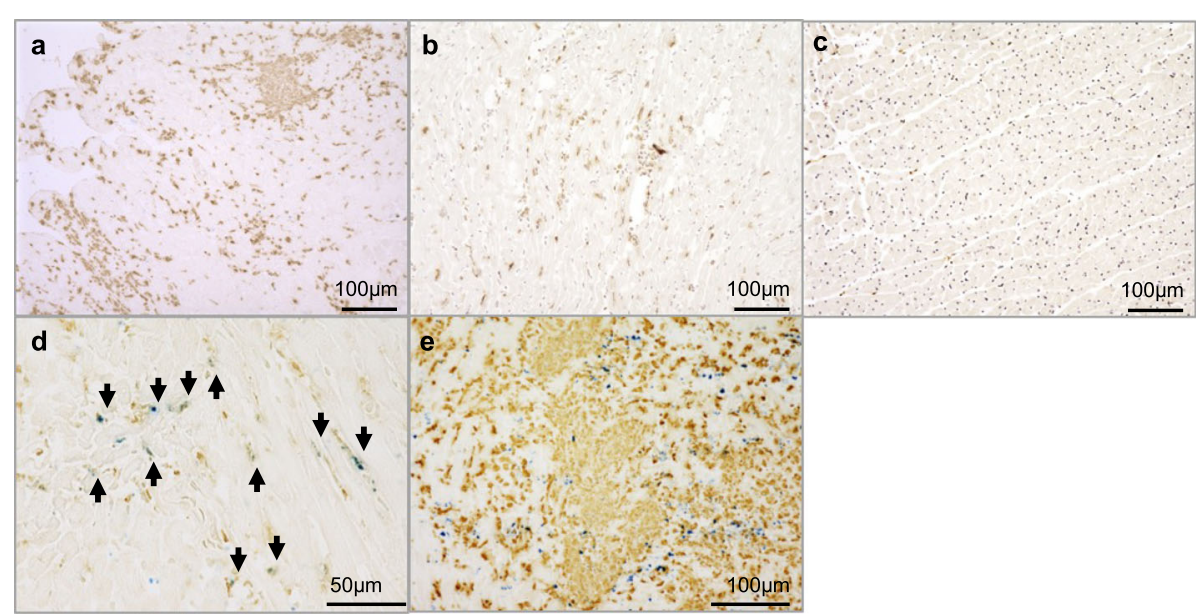

Fig. 6 Immunohistochemistry for macrophages Macrophage were detected as CD68 positive cells and visualized with DAB substrate. Macrophage infiltration in the myocardium was confirmed both at Day 21 (a) and Day 14 (b) but not in Control (c). Dual staining of iron particles (Prussian blue) and macrophages (DAB) shows their different distributions between $24 \mathrm{~h}(\mathbf{d})$ and $6 \mathrm{~h}(\mathbf{e})$ post-ferumoxytol injection

myocardial necrosis. FE-CMR is a promising diagnostic method to discriminate active myocardial inflammation at an early, potentially intervenable stage, which is superior to the detection/treatment window afforded by gadolinium-enhanced CMR.

This study also demonstrated that FE-CMR and T2* mapping at $6 \mathrm{~h}$ had a higher sensitivity to myocarditis compared to FE-CMR and T2* mapping at $24 \mathrm{~h}$. A recent clinical study also did not prove the superiority of $24 \mathrm{~h}$-FE-CMR over LGE in the diagnosis of acute myocarditis [30]. Imaging at the earlier time point following ferumoxytol injection could enhance the detection of inflammation. Because of the property of being taken up by phagocytes, images obtained at $24 \mathrm{~h}$ or later post injection of iron oxide particles have been widely used to provide functional information by detecting active inflammatory cells, dominated by macrophages, in inflammation-associated diseases including myocardial infarction, acute cardiac transplant rejection, arterial plaques, and tumors $[19,31,32]$. They have also shown the potential of a better sensitivity to smaller and less severe lesions compared to gadolinium-enhanced CMR [25, 32-34]. On the other hand, Yilmaz et al. detected signal loss in the infarct regions of acute myocardial infarction patients as early as $6 \mathrm{~h}$ post- ferumoxytol injection, which was explained by the phagocytotic activity of

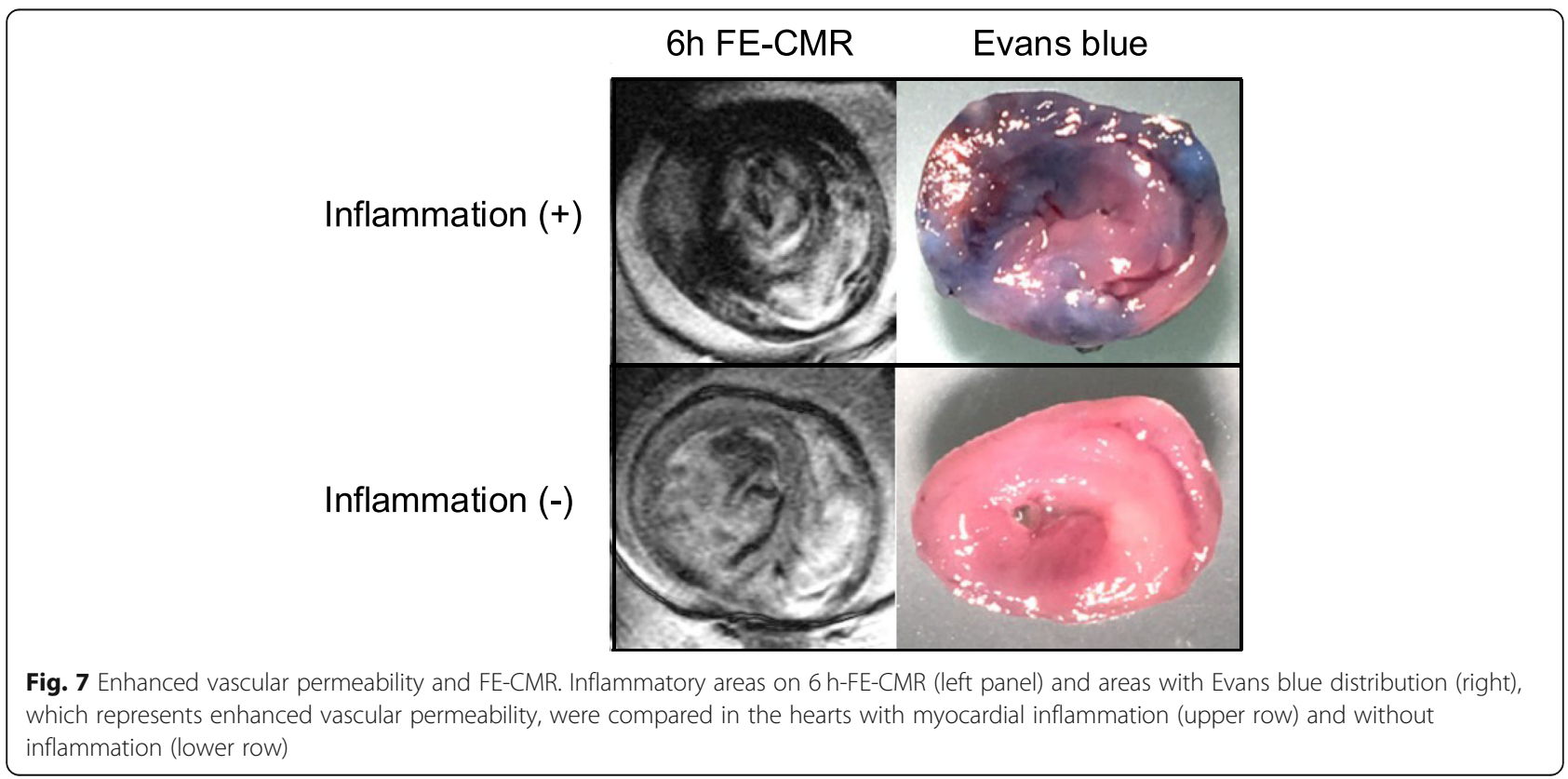


macrophages [21]. In addition, hyperemia and vascular leakage evoked by active inflammation could enhance the distribution of ferumoxytol in the inflammatory sites and promote the subsequent phagocytosis [16]. Ferumoxytol, originally developed as an intravascular contrast agent, has a relatively long plasma half-life time and persists in the blood for a long time. The dextran coating functions to slow the phagocytosis and release the iron from the core [35]. It has been used for contrast CMR angiography, cerebral blood volume mapping, and myocardial perfusion imaging [36-38]. Ferumoxytol is more suitable for enhancing hyperemia and vascular permeability than extravascular gadolinium-based contrast agents [39]. The inflammatory signals on $6 \mathrm{~h}$-FE-CMR observed in this study could have been exaggerated by dynamic enhancement of hyperemia and vascular leakage by ferumoxytol. The correspondence between inflammatory detection on $6 \mathrm{~h}$-FE-CMR and Evans Blue extravasation in our study supports this. High sensitivity of ferumoxytol to myocardial blood flow could also explain its less sensitivity to the tissue necrosis, which has poor blood supply as observed in our study.

Native T1, T2 and ECV mapping have been utilized for diagnosing myocarditis recently. A meta-analysis suggested higher sensitivity of native $\mathrm{T} 1$ compared to the Lake Louise Criteria, the current diagnostic criteria [40]. However, otherwise native T1, T2 and extracellular volume fraction (ECV) did not show significant improvement in sensitivity, specificity, or diagnostic odds ratio compared to Lake Louise Criteria. One of the drawbacks of native $\mathrm{T} 1$ is it reflects not only acute inflammatory change but also various chronic injuries including fibrosis or infiltrations. Mapping methods also need to be standardized to establish its diagnostic usefulness.

This study also showed correlation of increased precontrast $\mathrm{T} 2 *$ with advanced myocarditis. The area with high T2 corresponded with severe inflammation accompanying myocardial necrosis. In addition, the extent of myocardial necrosis had a positive correlation with myocardial T2\%. Although, the extent of fibrosis also showed a similar positive correlation with $\mathrm{T} 2 \%$, this was likely to be confounded by the linear correlation between the severity of necrosis and fibrosis at this phase of myocarditis. It is more reasonable that increased $\mathrm{T} 2 \%$ reflects necrosis rather than fibrosis, considering that necrosis is dominant at this phase and fibrosis, containing less water content, would be expected to decrease $\mathrm{T} 2^{*}$ [41]. $\mathrm{T} 2 *$ reflects both $\mathrm{T} 2$ and magnetic field heterogeneity, which is affected by the tissue microstructure [42]. Histologically, $\mathrm{T}^{*}$ is known to correlate with tissue water content and collagen fiber network, and the evaluation of $\mathrm{T} 2 *$ has been especially investigated in the diagnosis of cartilage degeneration [42]. The usability of T2* mapping in myocarditis remains unknown. A recent clinical study showed elevated $\mathrm{T} 2 *$ in the hypertrophic myocardium in the hypertrophic cardiomyopathy patients [43]. The increased T2* might result from the T2 change caused by myocardial edema. Because $\mathrm{T}^{*}$ decreases in the fibrotic tissue, $\mathrm{T}^{*}$ mapping could help to stage myocarditis by differentiating acute inflammation from post-myocarditis fibrotic replacement.

Although ferumoxytol has been already translated into clinical application, there have been no established protocols for the dose of ferumoxytol and the timing of imaging to detect inflammatory lesions. The dose of ferumoxytol used in this study $(16.8 \mathrm{mgF} / \mathrm{kg})$ was decided based on past animal studies and is higher than the clinical dose applied for perfusion or macrophage imaging (4-11 mgFe/ $\mathrm{kg}$ ). Clinical studies have shown higher doses of ferumoxytol improve image quality [44]. The dose and timing should be optimized in future studies.

\section{Limitation}

This study used a limited number of rats. Although myocarditis was induced in all rats, severity of infiltration and necrosis had variability among individuals. High heart rates of rats could potentially have led to underestimation of the diagnostic ability of gadoliniumenhanced CMR. T2w imaging is routinely used for diagnosis of myocarditis and FE-CMR needs to be compared with T2w imaging. We did not include T2w imaging in our study because of the insufficient image quality resulting from the high heart rate of the rats. However, T2w imaging suffers from inconsistency in diagnosing myocarditis in humans because of the shortcomings including subjective interpretation, low signal to noise ratio, low CNR, slow flow artifact, and variable signal intensity caused by the inversion recovery preparation [45]. Lastly, signal voids produced by ferumoxytol can mask the adjacent anatomic structure and the interpretation could be influenced by hemorrhage or artifacts. Alternative method such as positive contrast visualization of iron-oxide particles should be pursued in the future $[46,47]$.

\section{Conclusion}

FE-CMR acquired at $6 \mathrm{~h}$ enhance detection of early stages of myocarditis before development of necrosis or fibrosis. If this is proven in human clinical studies, it could potentially enable appropriate therapeutic intervention.

\section{Abbreviations}

BW: Body weight; CMR: Cardiovascular magnetic resonance; CNR: Contrast to noise ratio; ECG: Electrocardiogram; EGE: Early gadolinium enhancement; EPR: Enhanced vascular permeability and retention; FE: Ferumoxytolenhanced; GRE: Gradient echo; IR: Inversion recovery; LGE: Late gadolinium enhancement; LV: Left ventricle/left ventricular; LVEDV: Left ventricular enddiastolic volume; LVEF: Left ventricular ejection fraction; LVESV: Left ventricular end-systolic volume; LVM: Left ventricular mass; ROI: Region of 
interenst; SI: Signal intensity; SPIO: Superparamagnetic iron oxide; T2w: T2weighted; TE: Echo time; USPIO: Ultra-small SPIO

\section{Acknowledgements}

Tissue processing for histology and all staining were performed by Pauline Chu (histology lab, Stanford University School of Medicine). We greatly appreciate her excellent technical support.

\section{Author's contributions}

YT performed all animal studies, CMR studies, and image and histological analysis and prepared the manuscript. AT contributed to CMR studies and image analysis. SH contributed to manuscript preparation. PY contributed to image analysis. RD supervised research, helped image analysis and manuscript preparation. MM contributed to the study design, image interpretation and manuscript preparation. All authors read and approved the final Manuscript.

\section{Funding}

Kanae Foundation (YT), Japanese Circulation Society (YT),

\section{Availability of data and materials}

The datasets used and analyzed during the current study are available from the corresponding author on reasonable request.

\section{Ethics approval and consent to participate}

Animal procedures were reviewed and approved by both the Veterinary Service Center and Administrative Panel on Laboratory Animal Care (APLAC) to ensure that proposed procedures and anesthetics were appropriate. All aspects of pre- and post-procedural care were followed to ensure maximal survival by the rats. Procedures include physical examination, weighing the animals, supportive fluids, analgesics, daily body temperature, appetite/excretory functions, and care of surgical site were performed.

\section{Consent for publication}

Not applicable.

\section{Competing interests}

MVM is currently an employee of Google Health (Palo Alto, CA).

Received: 31 January 2019 Accepted: 21 November 2019

Published online: 16 December 2019

\section{References}

1. Herskowitz A, Campbell S, Deckers J, Kasper EK, Boehmer J, Hadian D, Neumann DA, Baughman KL. Demographic features and prevalence of idiopathic myocarditis in patients undergoing endomyocardial biopsy. Am J Cardiol. 1993;71:982-6.

2. Drory $Y$, Turetz $Y$, Hiss $Y$, Lev B, Fisman EZ, Pines A, Kramer MR. Sudden unexpected death in persons less than 40 years of age. Am J Cardiol. 1991; 68:1388-92.

3. Phillips M, Robinowitz M, Higgins JR, Boran KJ, Reed T, Virmani R. Sudden cardiac death in air force recruits. A 20-year review. JAMA. 1986;256:2696-9.

4. Rajs J, Hammarquist F. Sudden infant death in Stockholm. A forensic pathology study covering ten years. Acta Paediatr Scand. 1988;77:812-20.

5. Felker GM, Thompson RE, Hare JM, Hruban RH, Clemetson DE, Howard DL, Baughman KL, Kasper EK. Underlying causes and long-term survival in patients with initially unexplained cardiomyopathy. N Engl J Med. 2000;342: 1077-84.

6. Canter CE, Simpson KP. Diagnosis and treatment of myocarditis in children in the current era. Circulation. 2014;129:115-28.

7. Hufnagel G, Pankuweit S, Richter A, Schönian U, Maisch B. The European study of epidemiology and treatment of cardiac inflammatory diseases (ESETCID). First epidemiological results. Herz. 2000;25:279-85.

8. McMurray JJ, Adamopoulos S, Anker SD, Auricchio A, Böhm M, Dickstein K, Falk V, Filippatos G, Fonseca C, Gomez-Sanchez MA, et al. ESC guidelines for the diagnosis and treatment of acute and chronic heart failure 2012: the task force for the diagnosis and treatment of acute and chronic heart failure 2012 of the European Society of Cardiology. Developed in collaboration with the heart failure association (HFA) of the ESC. Eur Heart J. 2012;33: 1787-847.
9. Cooper LT, Baughman KL, Feldman AM, Frustaci A, Jessup M, Kuhl U, Levine GN, Narula J, Starling RC, Towbin J, et al. The role of endomyocardial biopsy in the management of cardiovascular disease: a scientific statement from the American Heart Association, the American College of Cardiology, and the European Society of Cardiology. Endorsed by the Heart Failure Society of America and the heart failure Association of the European Society of cardiology. J Am Coll Cardiol. 2007;50:1914-31.

10. Chow LH, Radio SJ, Sears TD, McManus BM. Insensitivity of right ventricular endomyocardial biopsy in the diagnosis of myocarditis. J Am Coll Cardiol. 1989;14:915-20.

11. Lynch JP, Hwang J, Bradfield J, Fishbein M, Shivkumar K, Tung R. Cardiac involvement in sarcoidosis: evolving concepts in diagnosis and treatment. Semin Respir Crit Care Med. 2014;35:372-90.

12. Simonetti OP, Finn JP, White RD, Laub G, Henry DA. "black blood" T2-weighted inversion-recovery MR imaging of the heart. Radiology. 1996;199:49-57.

13. Miller DD, Holmvang G, Gill JB, Dragotakes D, Kantor HL, Okada RD, Brady TJ. MRI detection of myocardial perfusion changes by gadolinium-DTPA infusion during dipyridamole hyperemia. Magn Reson Med. 1989;10:246-55.

14. Friedrich MG, Sechtem U, Schulz-Menger J, Holmvang G, Alakija P, Cooper LT, White JA, Abdel-Aty H, Gutberlet M, Prasad S, et al. Cardiovascular magnetic resonance in myocarditis: a JACC White paper. J Am Coll Cardiol. 2009;53:1475-87.

15. De Cobelli F, Pieroni M, Esposito A, Chimenti C, Belloni E, Mellone R, Canu T, Perseghin G, Gaudio C, Maseri A, et al. Delayed gadoliniumenhanced cardiac magnetic resonance in patients with chronic myocarditis presenting with heart failure or recurrent arrhythmias. J Am Coll Cardiol. 2006;47:1649-54

16. Podrouzkova H, Feitova V, Panovsky R, Meluzin J, Orban M. Superparamagnetic iron oxide-enhanced magnetic resonance for imaging cardiac inflammation. A minireview. Biomed Pap Med Fac Univ Palacky Olomouc Czech Repub. 2015;159:378-81.

17. Wang YX, Hussain SM, Krestin GP. Superparamagnetic iron oxide contrast agents: physicochemical characteristics and applications in MR imaging. Eur Radiol. 2001;11:2319-31.

18. Toth GB, Varallyay CG, Horvath A, Bashir MR, Choyke PL, Daldrup-Link HE, Dosa E, Finn JP, Gahramanov S, Harisinghani M, et al. Current and potential imaging applications of ferumoxytol for magnetic resonance imaging. Kidney Int. 2017;92:47-66.

19. Alam SR, Shah AS, Richards J, Lang NN, Barnes G, Joshi N, MacGillivray T, McKillop G, Mirsadraee S, Payne J, et al. Ultrasmall superparamagnetic particles of iron oxide in patients with acute myocardial infarction: early clinical experience. Circ Cardiovasc Imaging. 2012;5:559-65.

20. Richards JM, Semple SI, MacGillivray TJ, Gray C, Langrish JP, Williams M, Dweck M, Wallace W, McKillop G, Chalmers RT, et al. Abdominal aortic aneurysm growth predicted by uptake of ultrasmall superparamagnetic particles of iron oxide: a pilot study. Circ Cardiovasc Imaging. 2011;4:274-81.

21. Yilmaz A, Dengler MA, van der Kuip $H$, Yildiz H, Rösch S, Klumpp S, Klingel $K$, Kandolf R, Helluy X, Hiller KH, et al. Imaging of myocardial infarction using ultrasmall superparamagnetic iron oxide nanoparticles: a human study using a multi-parametric cardiovascular magnetic resonance imaging approach. Eur Heart J. 2013;34:462-75.

22. Huber SA. Autoimmunity in myocarditis: relevance of animal models. Clin Immunol Immunopathol. 1997;83:93-102.

23. Kodama M, Hanawa H, Saeki M, Hosono H, Inomata T, Suzuki K, Shibata A Rat dilated cardiomyopathy after autoimmune giant cell myocarditis. Circ Res. 1994;75:278-84.

24. Watanabe R, Azuma RW, Suzuki J, Ogawa M, Itai A, Hirata Y, Komuro I, Isobe M. Inhibition of NF-kB activation by a novel IKK inhibitor reduces the severity of experimental autoimmune myocarditis via suppression of T-cell activation. Am J Physiol Heart Circ Physiol. 2013;305:H1761-71.

25. Dousset V, Ballarino L, Delalande C, Coussemacq M, Canioni P, Petry KG, Caillé JM. Comparison of ultrasmall particles of iron oxide (USPIO)-enhanced T2-weighted, conventional T2-weighted, and gadolinium-enhanced T1weighted MR images in rats with experimental autoimmune encephalomyelitis. AJNR Am J Neuroradiol. 1999;20:223-7.

26. Dousset V, Gomez C, Petry KG, Delalande C, Caille JM. Dose and scanning delay using USPIO for central nervous system macrophage imaging. MAGMA. 1999:8:185-9.

27. Gilson WD, Kraitchman DL. Cardiac magnetic resonance imaging in small rodents using clinical 1.5 T and 3.0 T scanners. Methods. 2007;43:35-45.

28. Huang Z, Li C, Yang S, Xu J, Shen Y, Xie X, Dai Y, Lu H, Gong H, Sun A, et al. Magnetic resonance hypointensive signal primarily originates from extracellular 
iron particles in the long-term tracking of mesenchymal stem cells transplanted in the infarcted myocardium. Int J Nanomedicine. 2015;10:1679-90.

29. Brash JT, Ruhrberg C, Fantin A. Evaluating vascular Hyperpermeabilityinducing agents in the skin with the miles assay. J Vis Exp. 2018;136. https:// doi.org/10.3791/57524.

30. Stirrat CG, Alam SR, MacGillivray TJ, Gray CD, Dweck MR, Dibb K, Spath N, Payne JR, Prasad SK, Gardner RS, et al. Ferumoxytol-enhanced magnetic resonance imaging in acute myocarditis. Heart. 2017;104:300-5.

31. Bashir MR, Bhatti L, Marin D, Nelson RC. Emerging applications for ferumoxytol as a contrast agent in MRI. J Magn Reson Imaging. 2015;41:884-98.

32. Moon H, Park HE, Kang J, Lee H, Cheong C, Lim YT, Ihm SH, Seung KB, Jaffer FA, Narula J, et al. Noninvasive assessment of myocardial inflammation by cardiovascular magnetic resonance in a rat model of experimental autoimmune myocarditis. Circulation. 2012;125:2603-12.

33. Wu YL, Ye Q, Foley LM, Hitchens TK, Sato K, Williams JB, Ho C. In situ labeling of immune cells with iron oxide particles: an approach to detect organ rejection by cellular MRI. Proc Natl Acad Sci U S A. 2006;103:1852-7.

34. Xu S, Jordan EK, Brocke S, Bulte JW, Quigley L, Tresser N, Ostuni JL, Yang Y, McFarland HF, Frank JA. Study of relapsing remitting experimental allergic encephalomyelitis SJL mouse model using MION-46L enhanced in vivo MRI: early histopathological correlation. J Neurosci Res. 1998;52:549-58.

35. Finn JP, Nguyen KL, Han F, Zhou Z, Salusky I, Ayad I, Hu P. Cardiovascular MRI with ferumoxytol. Clin Radiol. 2016;71:796-806.

36. Hope MD, Hope TA, Zhu C, Faraji F, Haraldsson H, Ordovas KG, Saloner D. Vascular imaging with Ferumoxytol as a contrast agent. AJR Am J Roentgenol. 2015;205:W366-73.

37. Wu EX, Tang H, Wong KK, Wang J. Mapping cyclic change of regional myocardial blood volume using steady-state susceptibility effect of iron oxide nanoparticles. J Magn Reson Imaging. 2004;19:50-8.

38. D'Arceuil H, Coimbra A, Triano P, Dougherty M, Mello J, Moseley M, Glover $\mathrm{G}$, Lansberg M, Blankenberg F. Ferumoxytol enhanced resting state fMRI and relative cerebral blood volume mapping in normal human brain. Neuroimage. 2013;83:200-9.

39. Netto JP, Iliff J, Stanimirovic D, Krohn KA, Hamilton B, Varallyay C, Gahramanov S, Daldrup-Link H, d'Esterre C, Zlokovic B, et al. Neurovascular unit: basic and clinical imaging with emphasis on advantages of Ferumoxytol. Neurosurgery. 2017:82:770-80.

40. Pan JA, Lee YJ, Salerno M. Diagnostic performance of extracellular volume, native $\mathrm{T} 1$, and $\mathrm{T} 2$ mapping versus Lake Louise criteria by cardiac magnetic resonance for detection of acute myocarditis: a meta-analysis. Circ Cardiovasc Imaging. 2018;11:e007598.

41. van Nierop BJ, Bax NA, Nelissen JL, Arslan F, Motaal AG, de Graaf L, Zwanenburg JJ, Luijten PR, Nicolay K, Strijkers GJ. Assessment of myocardial fibrosis in mice using a $2^{*}$-weighted $3 \mathrm{D}$ radial magnetic resonance imaging sequence. PLoS One. 2015;10:e0129899.

42. Mamisch TC, Hughes T, Mosher TJ, Mueller C, Trattnig S, Boesch C, Welsch GH. T2 star relaxation times for assessment of articular cartilage at $3 \mathrm{~T}$ : a feasibility study. Skelet Radiol. 2012:41:287-92.

43. Huelnhagen T, Ku MC, Reimann HM, Serradas Duarte T, Pohlmann A, Flemming B, Seeliger E, Eichhorn C, A Ferrari V, Prothmann M, et al: Myocardial effective transverse relaxation time T. Sci Rep 2018, 8:3974.

44. Varallyay CG, Nesbit E, Fu R, Gahramanov S, Moloney B, Earl E, Muldoon LL, Li X, Rooney WD, Neuwelt EA. High-resolution steady-state cerebral blood volume maps in patients with central nervous system neoplasms using ferumoxytol, a superparamagnetic iron oxide nanoparticle. J Cereb Blood Flow Metab. 2013;33:780-6

45. Eitel I, Friedrich MG. T2-weighted cardiovascular magnetic resonance in acute cardiac disease. J Cardiovasc Magn Reson. 2011;13:13.

46. Stuber M, Gilson WD, Schär M, Kedziorek DA, Hofmann LV, Shah S, Vonken EJ, Bulte JW, Kraitchman DL. Positive contrast visualization of iron oxidelabeled stem cells using inversion-recovery with ON-resonant water suppression (IRON). Magn Reson Med. 2007;58:1072-7.

47. Korosoglou G, Weiss RG, Kedziorek DA, Walczak P, Gilson WD, Schär M, Sosnovik DE, Kraitchman DL, Boston RC, Bulte JW, et al. Noninvasive detection of macrophage-rich atherosclerotic plaque in hyperlipidemic rabbits using "positive contrast" magnetic resonance imaging. J Am Coll Cardiol. 2008;52:483-91.

\section{Publisher's Note}

Springer Nature remains neutral with regard to jurisdictional claims in published maps and institutional affiliations.

Ready to submit your research? Choose BMC and benefit from:

- fast, convenient online submission

- thorough peer review by experienced researchers in your field

- rapid publication on acceptance

- support for research data, including large and complex data types

- gold Open Access which fosters wider collaboration and increased citations

- maximum visibility for your research: over $100 \mathrm{M}$ website views per year

At BMC, research is always in progress.

Learn more biomedcentral.com/submissions 